\title{
A double-blind option for peer review
}

\section{Together with Nature and the other Nature-branded monthly journals, Nature Medicine is offering authors the option to remain anonymous during the peer-review process.}

$\mathbf{S}$ tarting in March, Nature and the monthly Nature-branded journals will experiment with an alternative to their timetested method of peer review. Instead of the traditional single-blind method, in which reviewers are anonymous but know the authors' identities, authors will be able to choose double-blind peer review, in which both authors and reviewers are unknown to each other.

Alternatives to the traditional single-blind peer-review process are often proposed. Chief among them are double-blind and open peer review, two apparent opposites, as in the latter both the authors and reviewers are known to each other. Not surprisingly, the reasons cited in favor of these two alternatives are different. On the one hand, proponents of open peer review see its transparency as a way to encourage more civil and thoughtful reviewer comments-although others are concerned that it promotes a less-critical attitude. On the other hand, advocates of double-blind peer review suggest that it eliminates ad hominem biases such as those based on gender, seniority, reputation and affiliation. Whether either method can meet these aspirations while also maintaining the necessary level of criticism remains a matter of debate.

Nature experimented with open peer review in 2006, but at the time, despite expressed interest, the uptake from both authors and reviewers was low and the open reviews were not technically substantive. Attitudes about open peer review are probably still evolving as several journals continue to experiment with variations on this practice. Opinions about double-blind review, however, are more consistent.

In one of the largest studies on peer review-a 2009 international and cross-disciplinary survey of more than 4,000 researchers (J. Am. Soc. Inf. Sci. Technol. 64, 132-161, 2013)$76 \%$ of respondents indicated that they believed that doubleblind peer review was an effective system (open and single-blind peer review were considered effective by $20 \%$ and $45 \%$ of respondents, respectively). Our own more-recent reader survey confirmed the desire to have double-blind peer review as an option, and this sentiment is echoed in conversations with young scientists worldwide. These discussions reveal a widespread perception that biases based on authorship affect traditional singleblind peer review, and they have contributed greatly to making us reconsider peer review options.
Many editors of Nature and the monthly Nature-branded journals have been skeptical about double-blind peer review. Some are uncertain about its efficacy, some are concerned about the potential difficulty of recruiting referees, and some see it as their responsibility to mitigate the biases that this system is intended to address. All editors take, and will continue to take, this responsibility seriously by maintaining awareness of any potential predispositions when selecting reviewers and considering reviewers' comments. They will also continue to honor reasonable requests to exclude particular reviewers, regardless of the chosen method of peer review. However, by definition, unconscious biases are difficult to identify and control. Several studies have detected involuntary biases, notably on the basis of gender, in other areas of the scientific enterprise, such as the hiring of laboratory staff, citation habits and speaker lineups at conferences. It is therefore difficult to guarantee a bias-free process.

Since June 2013, Nature Geoscience and Nature Climate Change have allowed authors to choose between double-blind and single-blind peer review at submission. The uptake of the double-blind method has been much lower than the enthusiasm expressed in surveys would have predicted: no more than a fifth of monthly submissions are going the double-blind route. Nevertheless, no substantial effects on the quality of reviews have been detected, and the reactions to this trial among surveyed authors have been sufficiently positive that Nature and the monthly Nature-branded journals have decided to join the experiment. (Nature Communications will join at a later date.)

The responsibility for rendering the manuscript anonymous falls to the authors. Clearly, in some situations, keeping an author's identity secret will be impossible because of awareness of his or her work in the specialist community. We will also continue to promote policies that support researchers who wish to release data early and discuss their work with their peers prior to publication, via conferences or preprint servers. Therefore, the double-blind process is optional on all titles. Some will choose it to assuage concerns about biases, others purely by principle.

We will keep this initiative under review, and we welcome further feedback from authors and referees. 